\title{
Transmission Electron Microscopy of 304-type Stainless Steel after Exposure to Neutron Flux and Irradiation Temperature Gradients
}

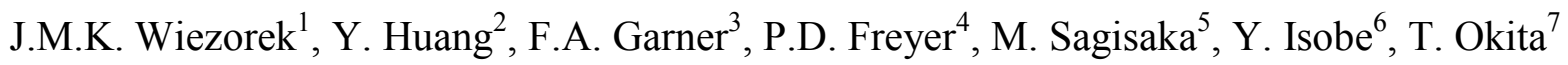 \\ ${ }^{1}$ Department of Mechanical Engineering and Materials Science, University of Pittsburgh, \\ Pittsburgh, PA, USA \\ 2. Department of Materials, University of Oxford, Oxford, UK \\ 3. Radiation Effects Consulting, Richland, WA, USA \\ 4. Westinghouse Electric Company LLC, Pittsburgh, PA, USA \\ 5. Westinghouse Electric Japan Ltd, Kobe, Japan \\ 6. Nuclear Fuels Industries Ltd, Osaka, Japan \\ 7. The University of Tokyo, Tokyo, Japan
}

Austenitic stainless steel (SS), such as grades 304 and 316, are used as structural materials in reactor internal components of light water cooled nuclear power plants and also find use in liquid metal cooled fast reactors. In the extreme environments of the nuclear reactors SS are degraded by exposure to neutron irradiation, leading to hardening and ductility loss and also to potentially significant distortion arising from void swelling, irradiation creep and irradiation assisted phase transformations. Prior studies of the degradation behavior and the underlying defect structure evolution related mechanisms in austenitic SS, have focused largely on examination of thin cross-section components, where effects from gradients in the neutron flux spectra and temperature are typically negligible. In contrast, for thick components with cross-sectional dimension of several centimeters, e.g. core and baffle plates in pressurized water reactors, which will receive neutron irradiation doses of up to $100 \mathrm{dpa}$ over 40 years of operational life [1], effects from neutron flux and $\gamma$-decay heating related thermal gradients can be significant. To address the paucity of experimental data regarding effects of thermal and neutron flux spectra gradients on the microstructural degradation in austenitic SS, we performed microstructural analyses by transmission electron microscopy (TEM) of 304-type SS reflector hex-blocks (Fig. 1a) from the EBR-II reactor. Depending on location within the reflector stack and within the hexblocks the 304-type SS experienced maximal irradiation dose of $\sim 33 \mathrm{dpa}$ and temperature gradients ranging from $\sim 378^{\circ} \mathrm{C}$ to $\sim 443^{\circ} \mathrm{C}$. Results from complementary non-destructive testing studies, e.g. locally resolved measurements of density changes and ultrasonic testing inspection, including the details of the procedures and schemes for extracting and preparing thin disk samples for TEM, have been reported recently [2]. The TEM analyses performed here determined a cold-deformed state for the reference material prior to irradiation (Fig. 1b). Dark field and bright field TEM imaging enabled a quantitative assessment of the variation in the volumetric void density, average void size, the numerical density and size of faulted dislocation loops, and the density of precipitates in the intragranular regions of the 304 SS microstructure in correlation to the gradients in neutron dose and temperature (e.g. Fig. 2). Two types of precipitates have been identified by selected area diffraction (SAD) to be consistent with a chemically ordered $\mathrm{L}_{2}$-structure $\gamma$ '-prime phase and cubic metal carbides of either $\mathrm{M}_{23} \mathrm{X}_{6}$ and/or $\mathrm{M}_{7} \mathrm{X}_{3}$ type, where $\mathrm{X}$ is predominantly $\mathrm{C}$ (Fig. 3). In addition, the TEM studies revealed an association of the decomposition of coarsened and irregular faulted Frank loops with the formation of cluster of nanoscale carbide precipitation in response to the neutron and thermal exposures. This would be consistent with radiation-induced segregation of alloying and impurity atoms to the strain fields of the dislocations and stacking faults of the Frank loops. Effects form the interplay of irradiation dose and temperature 
gradients, and implications for evaluation of degradation of thick section reactor internal components will be discussed.

\section{References}

[1] Kenik, E.A., Busby, J.T. , Mat. Sci. Eng. R (2012) 73: 67-83

[2] Etoh, J., et al., J. Nucl. Mat. (2013) 440: 500-507

[3] Support for the research from The Japanese Ministry of Education, Culture, Sports, Science and Technology, numerous staff members at the Westinghouse Materials Center of Excellence and Idaho National Laboratory are gratefully acknowledged.

a)

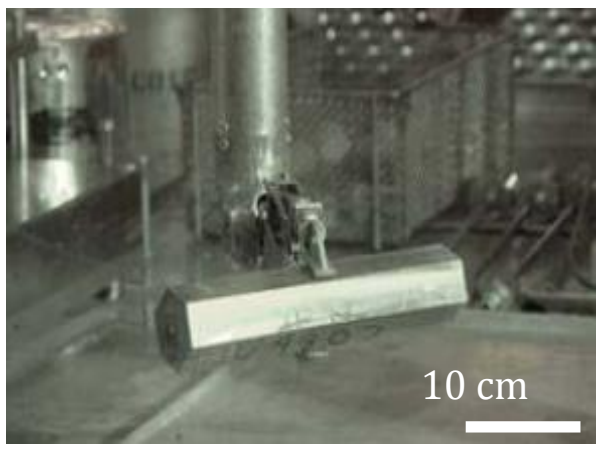

b)

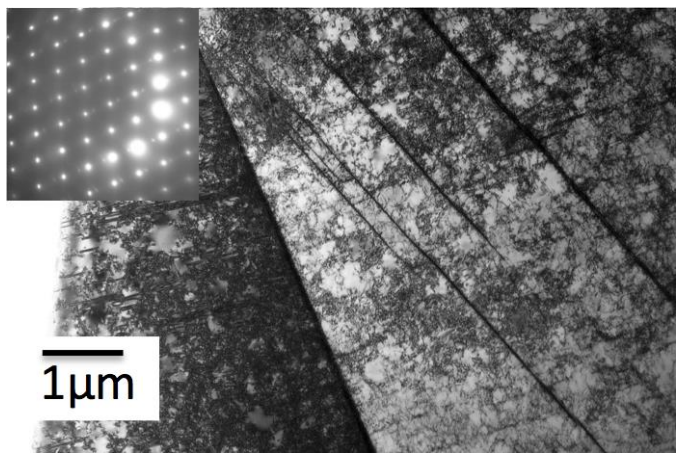

FIG. 1: a) EBR-II reflector hexblock (courtesy Idaho National Laboratory); b) TEM micrograph, inset SAD pattern, of cold-deformed reference 304 SS prior to irradiation.
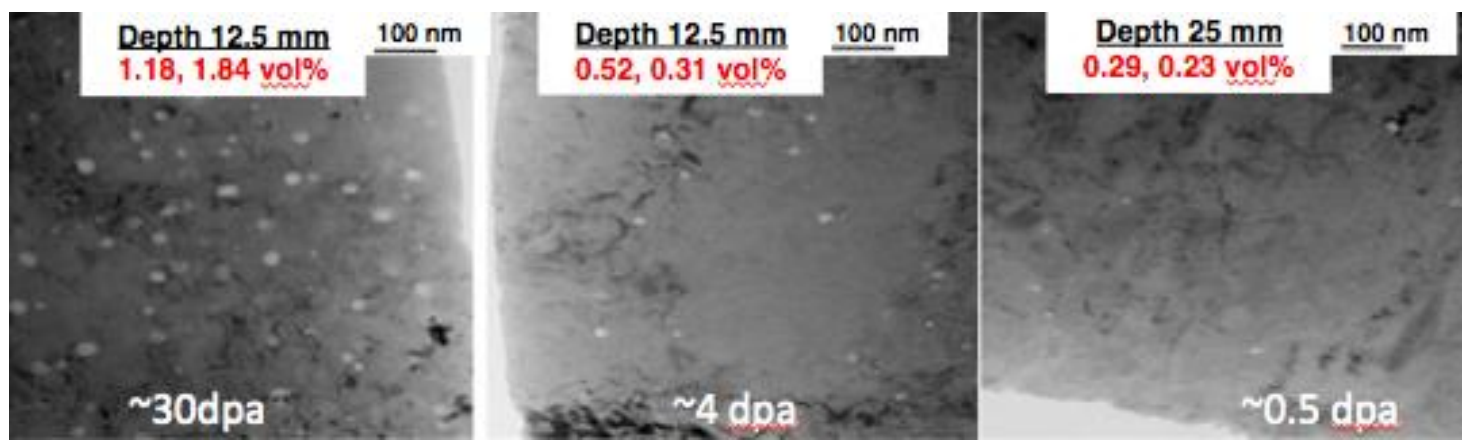

FIG. 2: Example TEM micrographs illustrating position dependent variations in volumetric swelling, void number density and void size with neutron dose.

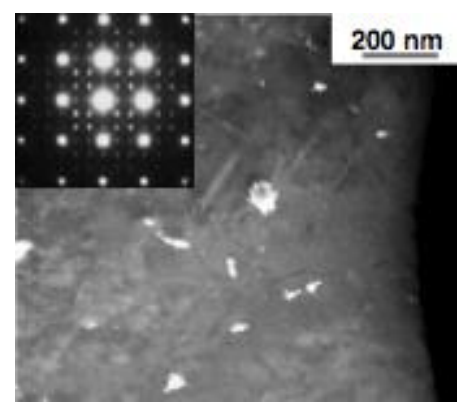

FIG. 3: Example dark field TEM image of precipitates formed in 304 SS EBR-II hexblock section after $\sim 0.5 \mathrm{dpa}$. Inset SAD pattern with prominent austenite matrix spots and precipitate diffraction maxima from two types of precipitates. Weaker precipitate maxima from $\gamma^{\prime}$-phase, Ni and Si enriched, and stronger precipitate maxima from larger number and volume density $\mathrm{M}_{23} \mathrm{X}_{6}$ and/or $\mathrm{M}_{7} \mathrm{X}_{3}$ type, $\mathrm{Cr}, \mathrm{C}$ and $\mathrm{Si}$ enriched, phase. 